\title{
Measurement System for Parameter Estimation and Diagnostic of Ultrasonic Transducers
}

\author{
P. Kluk*, A. Milewski, W. Kardyś, P. Kogut and P. Michalski \\ Tele and Radio Research Institute, Ratuszowa 11, 03-450 Warszawa, Poland
}

\begin{abstract}
The paper presents an architecture and design of measurement system and methods for parameter estimation and diagnostic of ultrasonic transducers used in ultrasonic welding and cutting systems manufactured by Tele and Radio Research Institute. The key element of the ultrasonic welding and cutting system is a high power ultrasonic transducer implemented as a sandwich transducer consisting of a stack of piezoelectric ceramic rings mounted between two masses. High quality welding and cutting demand predetermined frequency characteristic of transducer impedance. Also important are: high energy efficiency, high coupling coefficient, low dielectric loss, and the optimal radiation pattern. In order to manufacture high quality and long-life transducers the piezoelectric rings must be selected and the sandwich transducers diagnosed on the basis of their measured parameters. Presented measurement system takes advantage of the virtual instrument technique in the NI LabVIEW environment. It uses Agilent U2761A Function Generator, U2531A Data Acquisition Unit, and the linear amplifier to measure impedance frequency characteristic in the frequency range of $10 \mathrm{kHz}$ to $100 \mathrm{kHz}$. Moreover, the system can measure the vibration amplitude in the range of $1 \mu \mathrm{m}$ up to $100 \mu \mathrm{m}$, using an optical sensor, and the temperature of the transducer using a pyrometer sensor.
\end{abstract}

DOI: 10.12693/APhysPolA.124.468

PACS: 43.35.-c, 43.35.+d, 43.38.+n, 43.38.-p, 84.37.+q, 07.10.-h, 46.40.-f, 07.20.Dt

\section{Introduction}

The system enables automated measurement and diagnosis of high power piezoceramic transducers $[1,2]$, especially those used in ultrasonic welding and cutting systems. The low power subsystem equipped with the function generator, data acquisition module, and controlled force test fixture is designed for piezoceramic rings measurement and selection. The high power subsystem equipped with the $1200 \mathrm{VA}$ high power linear amplifier is intended for measurement and diagnosis of high power ultrasonic transducers and complete ultrasonic stacks. The vibration analysis subsystem equipped with the optical displacement sensor is used for sonotrode vibration analysis, especially for vibration amplitude measurement. The additional temperature measurement subsystem equipped with the thermal imaging camera and the pyrometer sensor is necessary because temperature generally has the main influence on measurements of piezoceramic transducers.

The system takes advantage of the virtual instrument technique in the NI LabVIEW environment. It uses Agilent U2761A Function Generator, U2531A Data Acquisition Unit, and the modified $1200 \mathrm{VA}$ acoustic linear amplifier for ultrasonic transducer electrical impedance measurement. Basing on the impedance frequency characteristic the RLC equivalent circuit parameter estimation is conducted. Additionally, selected piezoceramic material parameters can be estimated. Using sophisticated graphical user interface of the measurement sys-

*corresponding author; e-mail: piotr.kluk@itr.org.pl tem the impedance module and phase vs. frequency, and impedance circle can be displayed with resolution up to $0.1 \mathrm{~Hz}$ in the frequency range of $10 \mathrm{kHz}$ to $100 \mathrm{kHz}$. Vibration amplitude can be measured in the range of $1 \mu \mathrm{m}$ to $100 \mu \mathrm{m}$, with the resolution better than $1 \mu \mathrm{m}$, and visualized in the time and frequency domains. Sandwich transducer temperature map can be obtained using the thermal imaging camera. For a sandwich transducer closed in a metal housing the pyrometer sensor fixed in the hole in the housing must be used instead of the camera.

\section{Measurement methods \\ 2.1. Impedance measurement}

For piezoceramic rings $[1,2]$ the measurement circuit presented in Fig. 1 has been developed. The circuit is a modification of the one proposed in [3]. The complex electrical impedance $Z_{x}$ of the piezoceramic rings is evaluated using the following formula:

$$
Z_{x}=\frac{R_{p} U_{x}}{U_{p}-U_{x}} .
$$

The $W_{p}$ and $W_{x}$ voltage followers allow to minimize the influence of the U2531A input impedance (input capacitance mainly). The reference resistances $R_{p 1}=100 \Omega$ and $R_{p 2}=10 \mathrm{k} \Omega$ has been selected to provide approximately uniform distribution of uncertainty over the impedance absolute value measurement range of $1 \Omega$ to $1 \mathrm{M} \Omega$. The $R_{p}$ is automatically switched for $\left|Z_{x}\right|=$ $1 \mathrm{k} \Omega$ (with the $50 \Omega$ hysteresis). The controlled force test fixture equipped with a pneumatic actuator and a proportional precision pressure regulator provides the possibility to perform measurements with repeatable clamping force. 


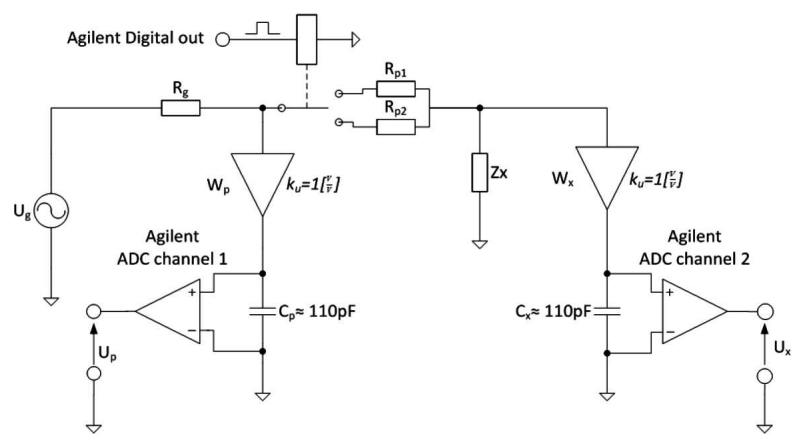

Fig. 1. Impedance measurement circuit of the low power subsystem.

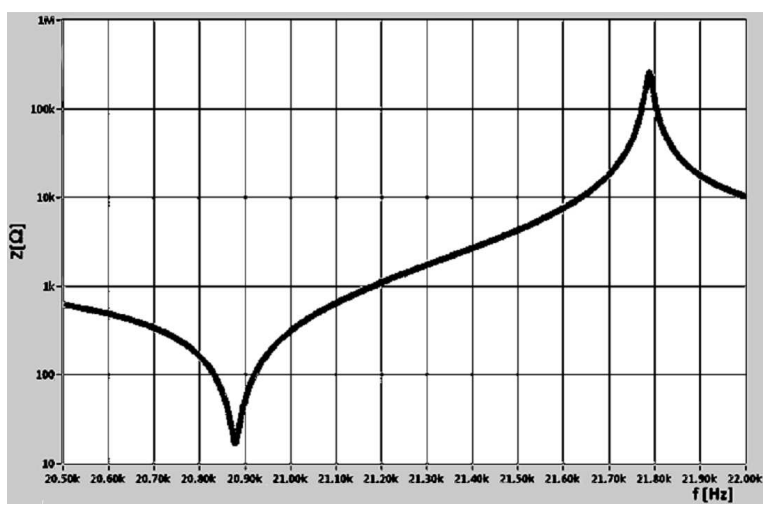

Fig. 2. Exemplary impedance module vs. frequency curve.

For high power transducers and complete ultrasonic stacks the function generator output signal is amplified using an acoustic amplifier equipped with a voltage step-up transformer. The voltage $U_{x}$ and current $I_{x}$ feeding the transducer are measured using the voltage and current transformers and then the impedance $Z_{x}$ is evaluated according to the well known formula

$$
Z_{x}=\frac{U_{x}}{I_{x}}
$$

Having the impedance $Z_{x}$ measured in the vicinity of the resonance and antiresonance frequencies, as showed in Fig. 2, the parameter identification of the ButterworthVan Dyke equivalent circuit (BVD model) $[1,2,4-6]$ is conducted.

\subsection{Vibrations measurement}

The vibrations analysis subsystem uses PHILTEC RC25-H3 fiber optic displacement sensor to measure: amplitude, velocity, and acceleration of the sonotrode vibrations. The output voltage signal of the sensor is directly proportional, with the sensitivity $S=9.2 \mathrm{mV} / \mu \mathrm{m}$, to the instantaneous distance between vibrating surface and the sensor, according to the calibration curve showed in Fig. 3. If the vibration is harmonic one, then the sensor output voltage signal $u_{p}(t)$ can be expressed in the following way:

$$
\begin{aligned}
& u_{p}(t)=S\left(A_{0}+A_{d} \sin (\omega t)\right)=S A_{0}+S A_{d} \sin (\omega t) \\
& \quad=U_{0}+U_{p} \sin (\omega t) .
\end{aligned}
$$

Consequently, the vibration amplitude $A_{d}$ can be estimated using the following formula:

$$
A_{d}=\frac{U_{p}}{S} \text {. }
$$

Generally, the high power ultrasonic systems are nonlinear [7], hence even for a pure sinusoidal excitation the ultrasonic stack response contains harmonics. Therefore, the Fourier analysis of the signal $u_{p}(t)$ should be conducted using the DFT transform [8]. Having the amplitude spectrum of the $u_{p}(t)$ signal we can compute vibration amplitude for the first harmonic using the formula (3). Analysis of other harmonics can be useful in the study of nonlinear phenomena occurring in high power ultrasonic systems. The velocity and acceleration can be simply computed by differentiating the $u_{p}(t)$ in the time domain.

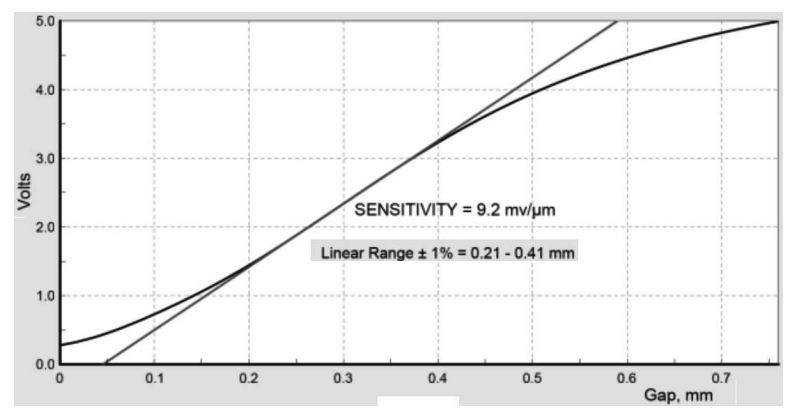

Fig. 3. PHILTEC RC25-H3 displacement sensor calibration curve.

\subsection{Temperature measurement}

Transducer temperature is measured at the side surface of the sandwich transducer in the range of $20^{\circ} \mathrm{C}$ to $100{ }^{\circ} \mathrm{C}$, with the resolution better than $1^{\circ} \mathrm{C}$ using the thermal imaging camera and the pyrometer sensor. Both calculate the temperature according to a well known Planck radiation law. The main measurement problem is to determine emissivity of the material because the amount of energy emitted by the object significantly depends on it. We are using the following procedure for determination of unknown emissivity. First assume the initial value $\varepsilon_{0}$ of emissivity $\varepsilon$ equal to 0.4 , then do the following:

a) Heat the transducer to the desired temperature $T_{0}$ with precision thermostat $\mathrm{PT} 200$;

b) Measure the temperature $T_{x}$ with the thermal camera or the pyrometer using the current emissivity value;

c) Calculate the absolute error $\Delta T$ of the measurement: $\Delta T=T_{0}-T_{x}$;

d) If $|\Delta T|<0.5^{\circ} \mathrm{C}$, then the determined value of emissivity $\varepsilon^{*}$ is equal to $\varepsilon_{i}$, and exit that calibration procedure;

e) If the error exceeds a predetermined value then: if $\Delta T<0$, then $\varepsilon_{i+1}=\varepsilon_{i}+0.02 ;$ if $\Delta T>0$, then $\varepsilon_{i+1}=$ $\varepsilon_{i}-0.02$

f) Go to step (a). 
The initial value of the emissivity was determined during measurements of PZT piezoceramic rings used in Tele and Radio Research Institute for manufacturing high power ultrasonic transducers. The emissivity of the ring side surface (polished ceramic with surface roughness about $1 \mu \mathrm{m}$ ) is between 0.3 and 0.5 . The step equal to 0.02 was selected arbitrarily to provide a compromise between the number of necessary steps and the required accuracy of determining the value of the emissivity.

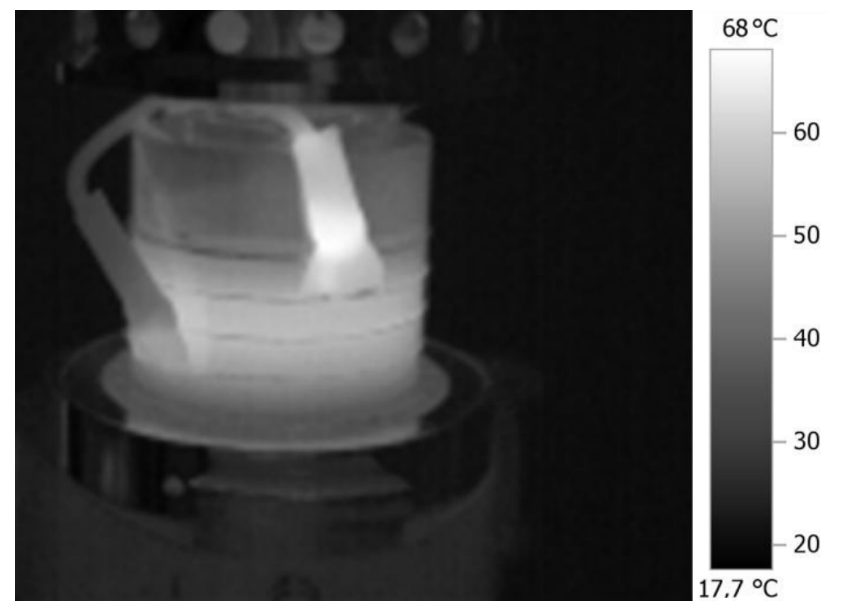

Fig. 4. Sandwich transducer temperature map.

The temperature map obtained from the thermal imaging camera is invaluable in the sandwich transducer diagnosis. For example, in Fig. 4 the sandwich transducer temperature map reveals a problem with one of its electrical connectors, where the temperature is significantly larger than at the other.

By measuring the sandwich transducer temperature by the pyrometer sensor mounted in the housing of the sandwich transducer along with the electric impedance characteristic of the transducer we can obtain impedance vs. temperature curves which are essential in diagnosis of high power ultrasonic transducers.

\section{Conclusions}

Presented measurement system has been successfully used for measurements and diagnosis of high power ultrasonic transducers and ultrasonic stacks. Using that system we can shape and verify the frequency response of the manufactured transducers and ultrasonic stacks. Having the reference impedance characteristic we can store it in an ultrasonic generator, and compare it with the actual one for the purpose of ultrasonic stack degradation monitoring. Moreover, the system has been successfully used for verification and validation of mathematical models of ultrasonic transducers. In the next step, we are going to use that system for examination and modeling of nonlinear phenomena occurring in high power ultrasonic systems.

\section{Acknowledgments}

We gratefully acknowledge the financial support of the National Science Centre, Poland, under grant number N N510 601840.

\section{References}

[1] M. Prokic, Piezoelectric Transducers Modeling and Characterization, MPI, Switzerland 2004.

[2] M.Đ. Radmanović, D.D. Mančić, Designing and Modelling of the Power Ultrasonic Transducers, MPI, Switzerland 2004.

[3] P. Kluk, P. Prystupiuk, Elektronika 49, 123 (2008) (in Polish).

[4] A. Arnau, Piezoelectric Transducers and Applications, Springer-Verlag, Berlin 2008.

[5] A. Milewski, P. Kluk, P. Kogut, J. FlorkowskaTrąbińska, Elektronika 52, 194 (2011) (in Polish).

[6] A. Milewski, P. Kogut, W. Kardyś, P. Kluk, Elektronika 53, 15 (2012) (in Polish).

[7] V.K. Astashev, V.I. Babitsky, Ultrasonic Processes and Machines - Dynamics, Control and Applications, Springer-Verlag, Berlin 2007.

[8] A.V. Oppenheim, R.W. Schafer, Discrete-Time Signal Processing, Prentice Hall, Upper Saddle River (NJ) 1998 